\title{
Lattice Location of Rare Earth Ions in Semiconductors: Interpretation and Limitations of using g values
}

\author{
David Carey \\ Nanoelectronics Centre, Advanced Technology Institute, University of Surrey, Guildford, \\ GU2 7XH, United Kingdom. \\ Email: David.Carey@ surrey.ac.uk
}

\begin{abstract}
The $g$ values of rare earth ions obtained from either paramagnetic resonance or Zeeman measurements are often used to interpret the location and/or environment surrounding rare earth ions. In the case of centres with cubic symmetry the $g$ value can be used to distinguish between substitutional and interstitial sites. For centres with less than cubic symmetry the average g value, taken as $1 / 3$ trace of the $g$ tensor, is often used as an indication of the lattice location and/or a measure of the strength of the local crystal field. This approach is widely used but is based on the assumption that the non-cubic terms in the total crystal field potential are small compared with the cubic crystal field. In this paper we have explored this assumption by calculating the principal $g$ values in axial crystal fields for the $\mathrm{Er}^{3+}$ ion. We examine the limits over which the average $g$ value approach is valid. Comparison is made with published results.
\end{abstract}

\section{INTRODUCTION}

Light emission from rare earth (RE) ions is now well established with emission from $\mathrm{Er}^{3+}$ ions being of particular importance since the transition between the two lowest spin-orbit energy levels occurs at the technologically important wavelength of $1.55 \mu \mathrm{m}$. Efficient luminescence can be assisted by maximising the concentration of optically active centres. It is now generally established that this is not the same as maximising the RE ion concentration since precipitation and/or up-conversion effects can dominate at higher RE ion concentrations [1]. Incorporation of light atoms, such as oxygen and carbon, have been shown to increase the solubility limit of Er in silicon through the formation of erbium impurity complexes [2]. The most appropriate method that can be used to elucidate the lattice location of the optically active RE ion is a crystal field analysis of high resolution photoluminescence (PL) spectra. In addition, the lattice location and/or coordination of RE ions (which includes both optically active and inactive ions) can be obtained from extended X-ray absorption fine structure [3], Zeeman measurements [4] and electron paramagnetic resonance measurements (EPR) [5]. The results from the latter two experiments are usually discussed in terms of $g$ values. In the case of Zeeman measurements, $g$ values are extracted on the basis of fitting PL peak positions to a suitable spin Hamiltonian. For EPR, the variation of the resonance magnetic field, as a function of orientation between the Zeeman applied field and the crystal axes is measured. Again by fitting the observed variation to a spin Hamiltonian, g values can be obtained which in turn can be interpreted. For systems with less than cubic symmetry various approaches and simplifications are used. It is the aim of this paper to test some of the commonly used approaches to determine the applicability and limitations used for the case of $\mathrm{Er}^{3+}$ ions in zincblende hosts. 


\section{RESULTS AND DISCUSSION}

\section{$\underline{\text { Cubic crystal field approximation }}$}

The $\mathrm{Er}^{3+}$ ion possesses eleven $4 f$ electrons which results in the lowest two spin-orbit ground states being ${ }^{4} \mathrm{I}_{15 / 2}$ (ground state) and ${ }^{4} \mathrm{I}_{13 / 2}$ (first excited state). Although the $4 f$ electrons are shielded from the full interaction of the host crystal field, the spin-orbit levels are nevertheless split into Stark levels, the number and symmetry of which are determined by the symmetry of the crystal field. In the case of cubic symmetry, group theory shows that the 16 -fold degenerate ${ }^{4} \mathrm{I}_{15 / 2}$ levels splits into five Stark levels labelled by the irreducible representations of the cubic group as $\Gamma_{6}+\Gamma_{7}+3 \Gamma_{8}$. Both $\Gamma_{6}$ and $\Gamma_{7}$ are doublets and each of the three $\Gamma_{8}$ are quartets. In the case of the ${ }^{4} \mathrm{I}_{13 / 2}$ state, a similar group theory analysis results in $2 \Gamma_{6}+\Gamma_{7}+2 \Gamma_{8}$. Which of the energy levels lies lowest is determined by the exact coordination and usually expressed in terms of the crystal field Hamiltonian given in Eq. (1) below

$$
H=B_{4}\left(O_{4}^{0}+5 O_{4}^{4}\right)+B_{6}\left(O_{6}^{0}-21 O_{6}^{4}\right)
$$

where $O_{\mathrm{m}}{ }^{\mathrm{n}}$ are the Steven's equivalent symmetry operators and $B_{4}$ and $B_{6}$ are the fourth and sixth order crystal field potentials, respectively. The solution to Eq. (1) is usually calculated by expressing the Hamiltonian in terms of a dimensionless variable $x$ (a measure of $\mathrm{B}_{4} / \mathrm{B}_{6}$ ) which runs between -1 and +1 and energy scale parameter $W$. For the ${ }^{4} \mathrm{I}_{15 / 2}$ level, and with labelling appropriate to $\mathrm{T}_{\mathrm{d}}$ symmetry, with $W>0$, the $\Gamma_{7}$ level will lie lowest between $-1<x<-0.46, \Gamma_{6}$ level will lie lowest between $-0.46<x<0.58$ and for $x>0.58$ the lowest energy state will be $\Gamma_{8}$. The spacing between the energy levels is controlled by $W$.

From the diagonalisation of the Hamiltonian in Eq. (1), the resultant crystal field eigenstates can be used to calculate the principal $g$ values. For the case of the $\Gamma_{6}$ state the $g$ value is 6.8 and for $\Gamma_{7}$ state the $g$ value is 6.0. In both cases the $g$ value is independent of $x$. The case of the lowest lying $\Gamma_{8}$ state is slightly more complicated, not because the spin Hamiltonian for a quartet is especially difficult, though additional terms may be need to be included, but the $g$ value is now dependent on the value of $x$. Such a situation arises when the irreducible representation of the same symmetry appears more than once. The interpretation given to the value of $x$ is also an important consideration. For the case of $\mathrm{Er}^{3+}$ ion in zincblende lattice, positive values of $x$ are interpreted as implying that the RE ion is located at a tetrahedral interstitial site $\left(T_{i}\right)$. Negative values of $x$ suggest that the ion is located at a substitutional site $\left(\mathrm{T}_{\mathrm{s}}\right)$. Such interpretations are based on knowledge of both the nearest neighbour (nn) and next nearest neighbour (nnn) atoms. In both $T_{i}$ and $T_{\mathrm{s}}$ sites the nn atoms lie $\sqrt{3} a / 4$ from the RE ion in equivalent $\langle 111\rangle$ directions, however, in the $\mathrm{T}_{\mathrm{s}}$ site the $12 \mathrm{nnn}$ atoms lying in $\langle 110\rangle$ directions are at a distance of $a / \sqrt{2},(a$ being the lattice constant). This differs from the case of $T_{i}$ site in which the $6 \mathrm{nnn}$ atoms lying in the $\langle 100\rangle$ directions are at a distance of $a / 2$. This is only $15 \%$ further out than the nn atoms and it is the octahedral field from these $6 \mathrm{nnn}$ atoms that results in the positive value of $x$.

For the case of $\mathrm{Er}^{3+}$ centres with less than cubic symmetry, analysis of the g values is usually performed by examination of the average of the trace of the $g$ value tensor, $g_{a v}$, with the $g$ values found in cubic symmetry where $g_{a v}$ is

$$
g_{a v}=1 / 3\left(g_{1}+g_{2}+g_{3}\right) .
$$


This analysis is performed assuming that the total crystal field is dominated by the 'parent' cubic potential. The lower symmetry crystal field terms can then be considered as a perturbation to the cubic terms. In general, the average $g$ value is often found to be slightly less than predicted for a $\Gamma_{6}$ or $\Gamma_{7}$ state. Such deviations are usually attributed to covalency effects and quantified using the orbital reduction factor $(1-k)$. Values of $(1-k)$ for different ions are given in table 1 below.

Table 1. Value of orbital reduction factor for a variety of hosts

\begin{tabular}{|l|c|c|}
\hline Ion and Host & $(1-k)$ & Ref. \\
\hline $\mathrm{Er}^{3+}$ in $\mathrm{ErBa}_{2} \mathrm{Cu}_{3} \mathrm{O}_{7-\delta}$ & 0.02 & 6 \\
\hline $\mathrm{Yb}^{3+}$ in $\mathrm{CaO}$ & 0.02 & 7 \\
\hline $\mathrm{Tm}^{2+}$ in $\mathrm{CaF}_{2}$ & 0.01 & 7 \\
\hline $\mathrm{Fe}^{3+}$ in $\mathrm{MgO}$ & 0.20 & 7 \\
\hline $\mathrm{Ti}^{3+}$ in $\mathrm{Al}_{2} \mathrm{O}_{3}$ & 0.30 & 7 \\
\hline $\mathrm{Er}^{3+}$ in $\mathrm{Si}$ & 0.12 & 5 \\
\hline
\end{tabular}

It is clear from the first three rows of Table 1 that the orbital reduction factors (ORFs) for RE ions are small $(<0.03)$ but that significantly larger ORFs are found with ions from the first row of the transition series where crystal field effects are stronger. The final value of $(1-k)$ for the case of Er codoped with $\mathrm{O}$ in $\mathrm{Si}$ is the value that would be required to explain the average $\mathrm{g}$ value (=6.28) obtained from low temperature EPR measurements [5] if the corresponding cubic energy level has a g value of 6.8. Such a large ORF is not considered realistic for RE ions and large observed shifts away from predicted g values cannot be explained solely by covalency effects.

For the case of $\mathrm{Er}^{3+}$ in axial symmetry, both the $\Gamma_{6}$ and $\Gamma_{7}$ cubic levels remain as doublets and the cubic $\Gamma_{8}$ levels each split into two doublets. For the case of a small axial trigonal crystal field superimposed on the cubic field, variation of the principal $g$ values $g_{\|}$and $g_{\perp}$ can be seen in Fig. 1 below. Two sets of data are presented corresponding to two of three possible $\Gamma_{8}$ lying as the ground state.

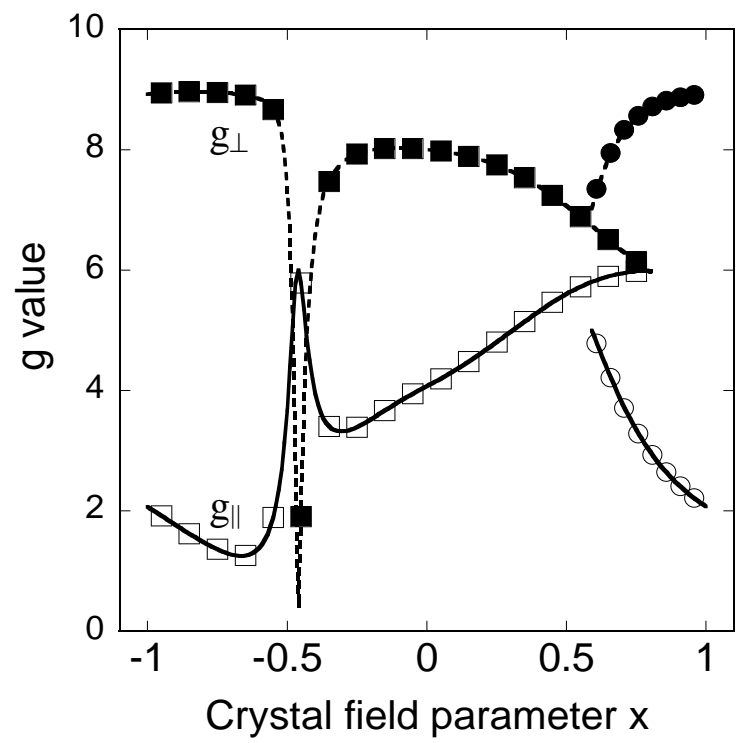

Figure 1 Variation of $\mathrm{g}_{\|}$(open symbols) and $\mathrm{g}_{\perp}($ closed) in a trigonal crystal field. Squares correspond to a $\Gamma_{8}$ level lying lowest with $\mathrm{W}<0$ and circles for a $\Gamma_{8}$ level lying lowest with $\mathrm{W}>0$. 
For the case of axial $\mathrm{Er}^{3+}$ centres derived from $\Gamma_{6}$ or $\Gamma_{7}$ states, the average $\mathrm{g}$ value should be equal to 6.8 or 6.0 , respectively. The solid lines in Fig. 2 shows how $g_{\|}$and $g_{\perp}$ must vary in order to satisfy this condition. Also included in Fig. 2 are $\mathrm{g}$ values for a number of $\mathrm{Er}^{3+}$ centres taken from the literature [8].

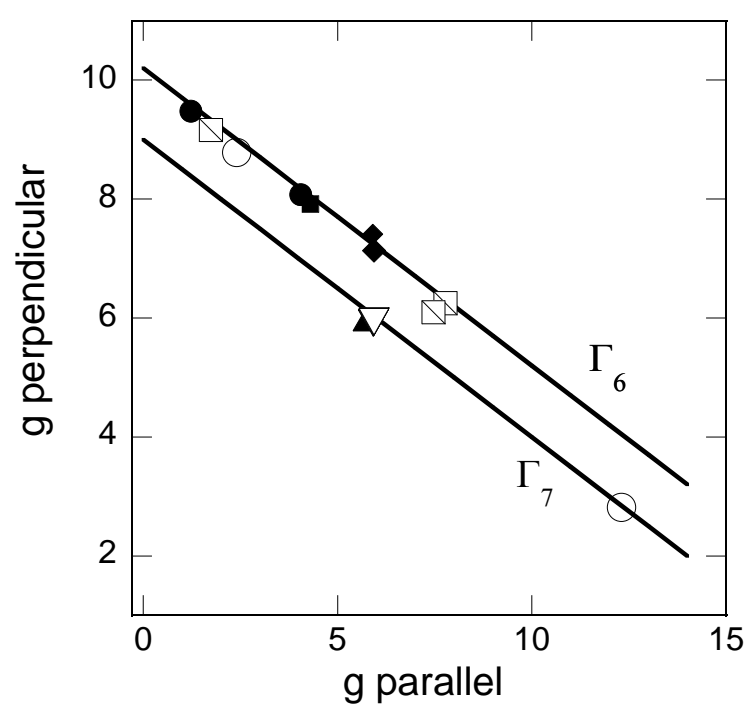

Figure 2 Solid lines show the variation of $g_{\perp}$ with $g_{\|}$for $\mathrm{Er}^{3+}$ assuming either $\Gamma_{6}$ or $\Gamma_{7}$ ground state. Symbols are experimentally measured values taken from the literature, summarised in reference 8 , in a range of hosts.

Figure 2 shows that from a range of samples the predicted $g$ values appear to follow the pattern expected by lying on either the line associated with $\Gamma_{6}$ or $\Gamma_{7}$ states. However, there is a greater tendency for more data points to lie on the line associated with $\Gamma_{6}$ state corresponding to an average $g$ value of 6.8 .

\section{$\underline{\text { Non-cubic crystal field approximation }}$}

In order to test the validity of the predominantly cubic crystal field approximation as an explanation of the observed $g$ value, an axial crystal field (of the form $\mathrm{B}_{2}{ }^{0} \mathrm{O}_{2}{ }^{0}$ ) was superimposed on the Hamiltonian in Eq. (1). Shown in Fig. 3(a) below is the variation of the principal $g$ values for a $\Gamma_{7}$ ground state over a wide range of axial crystal fields. It can first be observed that in the region where $\mathrm{g}_{\|}$(open circles) is high (above 10) the value of $\mathrm{g}_{\perp}$ is approaches zero. Indeed the value of $\mathrm{g}_{\perp}$ is below 2 for $\mathrm{B}_{2}{ }^{0}<-16 \mathrm{~cm}^{-1}$. Since the intensity of the EPR transition is governed by the strength of the matrix element associated with $g_{\perp}$, these transitions will not normally be observable. We believe that this explains the sparse number of data points at high values of $g_{\|}$in Fig. 2. For $\mathrm{B}_{2}{ }^{0}<30 \mathrm{~cm}^{-1}$, the average $\mathrm{g}$ value is about $6.0 \pm 0.1$, as observed in Fig. 3(b). Since $\mathrm{g}_{\|}$is less than zero when $\mathrm{B}_{2}{ }^{0}>30 \mathrm{~cm}^{-1}$, Fig. 3(b) shows the average $\mathrm{g}$ value for both real (i.e. negative) and absolute values of $g_{\|}$. This is an important consideration since absolute value of $g_{\|}$ is usually measured in an experiment. 

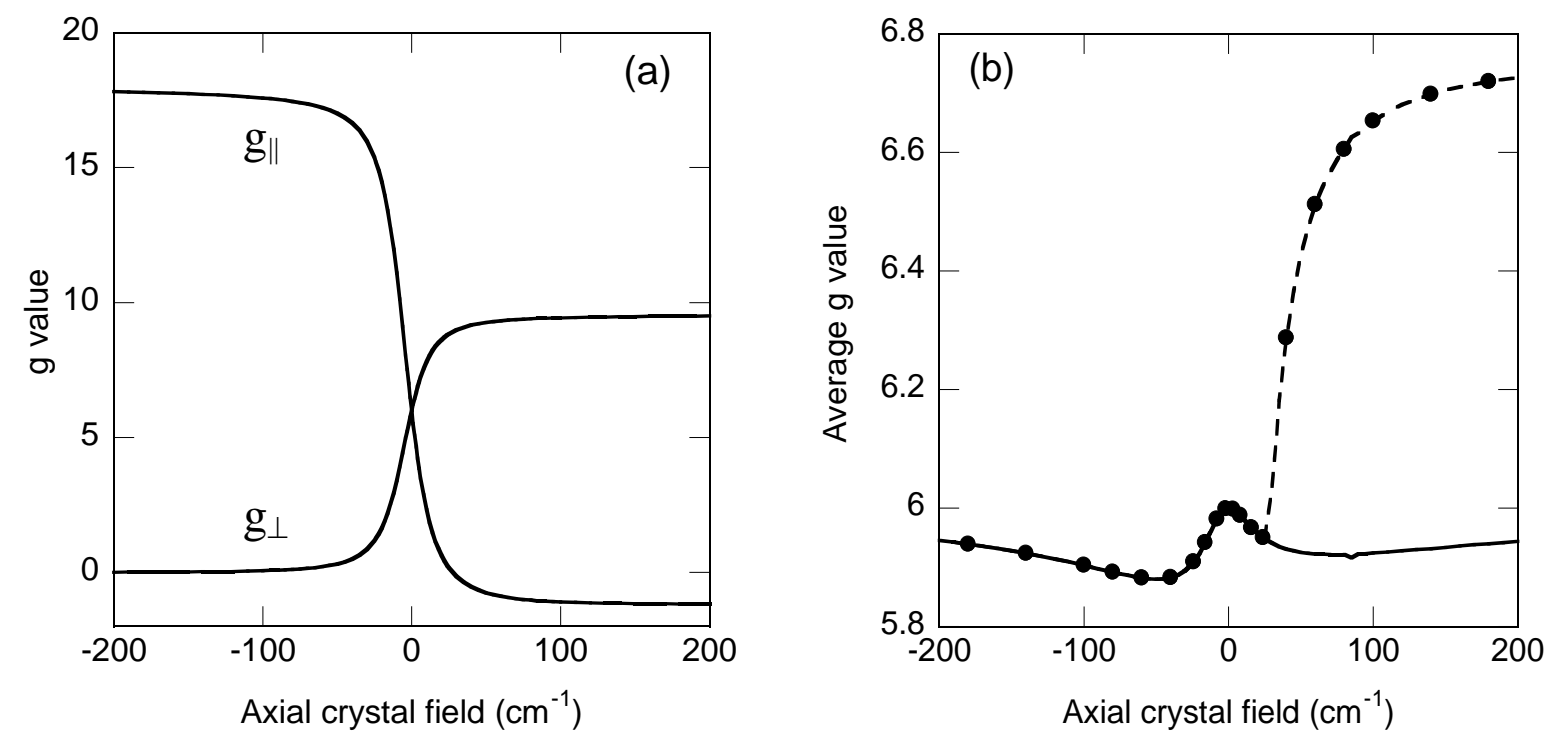

Figure 3 (a) Variation of $g_{\|}$and $g_{\perp}$ for $\operatorname{Er}^{3+}$ assuming $\Gamma_{7}$ ground state. (b) Variation of average $g$ value calculated from Fig. 3(a) assuming that the real (i.e. negative) value of $g_{\|}$is used (solid line) or the absolute value of $\mathrm{g}_{\|}$is used (dashed line and closed symbols).

For $\mathrm{B}_{2}{ }^{0}>30 \mathrm{~cm}^{-1}$, Fig. 3(b) shows that the average $\mathrm{g}$ value begins to deviate from that predicted by assuming the cubic crystal field dominates if the absolute value of $\mathrm{g}_{\|}$is used. For an axial field with $\mathrm{B}_{2}^{0}=60 \mathrm{~cm}^{-1}, \mathrm{~g}_{\mathrm{av}}=6.51$ and at high axial fields strengths $\left(\sim 150 \mathrm{~cm}^{-1}\right), \mathrm{g}_{\mathrm{av}}$ reaches 6.70 before leveling off. It is in this regime that it may appear that $\mathrm{g}$ value is now reminiscent of that associated with a $\Gamma_{6}$ state, rather than $\Gamma_{7}$. When the real value is used in its place $g_{a v}$ stays within 0.1 of what would be predicted. As a result care must be employed when interpreting the average $\mathrm{g}$ value in this case.
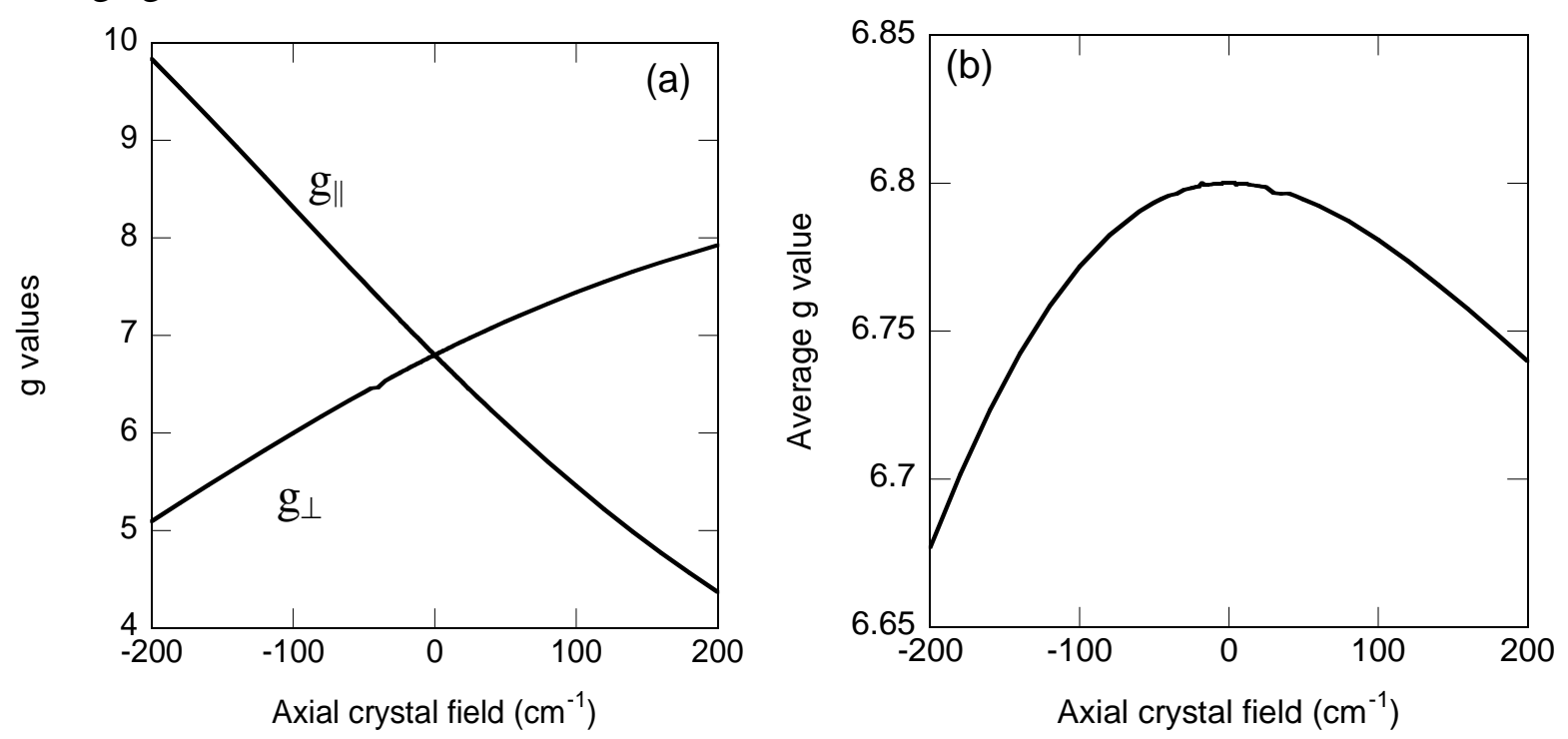

Figure 4 (a) Variation of $g_{\|}$and $g_{\perp}$ for $\operatorname{Er}^{3+}$ assuming $\Gamma_{6}$ ground state. (b) Variation of average $g$ value calculated from Fig. 4(a). 
No significant change of the principal $g$ values is observed (Fig. 4(a)) for the $\Gamma_{6}$ ground state and as a result the average $g$ value does not deviate significantly from 6.8 over the wide range of axial fields that have been used. Over the course of $\pm 200 \mathrm{~cm}^{-1}$ variation in the axial field, the average $g$ value remains within 0.1 of the predicted 6.8 for a $\Gamma_{6}$ ground state as shown in (Fig. 4(b)).

Finally, we note that for the $\Gamma_{6}$ state the magnitude of the $g$ value difference, $\left|\mathrm{g}_{\|}-\mathrm{g}_{\perp}\right|$, scales approximately linearly with axial field up to $200 \mathrm{~cm}^{-1}$ with a slope of $0.02 / \mathrm{cm}^{-1}$. As such the $\mid \mathrm{g}_{\|}$ $\mathrm{g}_{\perp} \mid$ may be used as a quantitative measure of the strength of the axial field. However, we do not find such a linear relationship for the $\Gamma_{7}$ state.

\section{CONCLUSIONS}

In this paper we have calculated the principal $\mathrm{g}$ values associated with an $\mathrm{Er}^{3+}$ centre with axial symmetry with different possible ground states. We have investigated the well known approach of using the average $g$ value as an indication of the ground state. These results show that the use of the average $g$ value is acceptable over a wide range of axial field strengths for a Stark level with a $\Gamma_{6}$ ground state. However, the approximation may lead to incorrect assumptions for a $\Gamma_{7}$ ground state in the presence of strong axial fields where the value of $g_{\|}$is less than zero. As a consequence care must be considered if the correct assignment is Stark energy level is to be made. The effects of covalency have been discussed and any observed large difference between $g_{a v}$ and the $g$ value predicted from cubic symmetry are unlikely to be due to covalency effects.

\section{ACKNOWLEDGEMENTS}

DC acknowledges the EPSRC for funding via an Advanced Research Fellowship.

\section{REFERENCES}

1. see, for example, MRS Proceedings on light emission from silicon.

2. F. Priolo, G. Franzò, S. Coffa, A. Polman, S. Libertino, R. C. Barklie and D. Carey, J. Appl. Phys. 78, 3874 (1995).

3. A. Terrasi, G. Franzò, S. Coffa, F. Priolo, F. D’Acapito and S. Mobilio, Appl. Phys. Lett. 70 $1712(1997)$

4. N.Q. Vinh, H. Przybylinska, Z.F. Krasil'nik and T. Gregorkiewicz, Phys. Rev. Lett. 90, $066401(2003)$

5 J. D. Carey, R. C. Barklie, J. F. Donegan, F. Priolo, G. Franzò and S. Coffa, Phys. Rev. B 59, 2773 (1999).

6. S.-Y. Wu and H.-N. Dong, J. Alloys and Compounds 379, 36 (2004).

7. values quoted from A. Abragam and B. Bleaney Electron Paramagnetic Resonance Ions (Oxford: Oxford University Press, 1970)

8. C.A.J. Ammerlann and I. De Maat-Gersdorf, Appl. Magn. Reson. 21, 13 (2001). 